\title{
Effect of STK17A on the sensitivity of ovarian cancer cells to paclitaxel and carboplatin
}

\author{
JIANHUA GAO, DAN LIU, JIE LI, QIANLIN SONG and QI WANG \\ Department of Obstetrics and Gynecology, The First Affiliated Hospital of Harbin Medical University, \\ Harbin, Heilongjiang 150001, P.R. China
}

Received March 16, 2015; Accepted April 25, 2016

DOI: $10.3892 / \mathrm{ol} .2016 .4727$

\begin{abstract}
Ovarian cancer is the main cause of cancer mortality in gynecological tumors around the world. Drug resistance to a variety of chemotherapeutics continue to be one of the main causes of treatment failure. In a previous study, it was demonstrated that STK17A, a proapoptotic gene, was significantly downregulated in acquired resistance phenotypes of colon cancer cells that are resistant to oxaliplatin and 5-fluorouracil. Therefore in the present study, the association between STK17A expression and ovarian cancer with initial drug resistance was investigated and the influence of STK17 on ovarian cancer cell proliferation and doubling time. In the present study, ovarian cancer cell lines that express low levels of STK17A were established by targeting STK17A with specific siRNA. In addition, up-regulation of STK17A was established in ovarian cells by pCDNA3flu/STK17A. The sensitivity of the transfected cells and controls to paclitaxel, carboplatin was examined by MTT assay, and the levels of proliferation and apoptosis were analyzed by flow cytometry. In the cells that were transfected with siRNA resulting in reduced expression of STK17A, the $50 \%$ inhibitory concentration $\left(\mathrm{IC}_{50}\right)$ of the chemotherapy drugs paclitaxel and carboplatin was increased compared with control cells $(\mathrm{P}<0.05)$. By contrast, in the cells that overexpressed STK17A following treatment with pCDNA3flu/STK17A, the $\mathrm{IC}_{50}$ of the chemotherapy drugs reduced in each case, and was significantly lower compared with the control $(\mathrm{P}<0.05)$. There was a variable susceptibility to carboplatin and paclitaxel resulting from altering the levels of STK17A expression in ovarian cancer cell lines. The growth of STK17A/siRNA transfected cells was promoted compared with that of the control cells and accordingly their cell doubling time was shortened.
\end{abstract}

Correspondence to: Professor Jianhua Gao, Department of Obstetrics and Gynecology, The First Affiliated Hospital of Harbin Medical University, 23 Post Street, Harbin, Heilongjiang 150001, P.R. China

E-mail: 1ilyjianhua@163.com

Key words: STK17A, ovarian cancer, cell cycle, drug-resistance MTT assay, flow cytometry

\section{Introduction}

At present, ovarian cancer is one of the main causes of mortality in gynecologic tumors around the world (1). Further, a variety of chemotherapeutic drug resistance continue to be the primary cause of treatment failure (2). Reduced drug accumulation, enhanced detoxification capability, aberrant apoptosis pathway, and increased repair of drug-induced DNA damage are the primary factors contributing to tumor cells resistance phenotypes $(3,4)$. However no biomarkers have been identified that have achieved the necessary specificity to predict the development of initial drug resistance in ovarian cancer. This has prompted studies to investigate the cellular and molecular mechanisms of drug resistance in ovarian cancer cells.

In a previous study, Tang et al (5) demonstrated that STK17A, one of the proapoptotic genes was significantly downregulated in acquired resistance phenotypes of colon cancer cells that are resistant to oxaliplatin and 5-fluorouracil. Wittig et al (6) also confirmed reduced levels of STK17A expression in the MeWoEtol cell line with acquired resistance to the DNA-damaging agent etoposide and determined that STK17A was located on chromosome $7 \mathrm{p}$ and that this region has undergone a loss in this sub-line. Whether down-regulation the level of STK17A expression is associated with drug resistance, or leading to cell aberrations that is uncertain. Therefore, in order to study the association between STK17A and initial drug resistance in ovarian cancer, ovarian cancer cell lines were established that express low levels of STK17A via targeted siRNA, and ovarian cancer cells were transfected with a vector encoding STK17A cDNA to up-regulate STK17A expression.

STK17A is a serine/threonine kinase, also been termed as death associated protein kinase (DAPK)-related apoptosis-inducing protein kinase (DRAK) 1, primarily functions to mediate apoptosis by associating with DAPK $(7,8)$. It is exclusively localized to the nucleus, and is expressed at the highest levels in the placenta (8). This molecule has recently been identified to be downregulated in cancer cells with acquired drug resistance. However, it is uncertain whether STK17A is a causative factor in ovarian cancer, or just a involved in the changes in tumor cells. The present study aimed to determine the effects of different levels of STK17A expression in the ovarian cancer cell line OVCAR3, and whether its 
expression affects cellular susceptibility to paclitaxel and the DNA-damaging drug, carboplatin.

\section{Materials and methods}

Cell line and cell culture. The ovarian cancercell line, OVCAR3, was obtained from the TianJin Life Science Research Center (TianJin, China). OVCAR3 cells were cultured in containing 10\% fetal bovine serum (FBS; Sigma Chemical, St. Louis, MO, USA), $100 \mathrm{u} / \mathrm{ml}$ penicillin, $100 \mathrm{mu} \mathrm{g} / \mathrm{ml}$ of streptomycin (GIBCO, ThermoFisher Scientific, Inc., Waltham, MA, USA) MEM $\alpha$ medium (Promega Corporation, Madison, WI, USA) and incubated in a humidified atmosphere containing $5 \% \mathrm{CO}_{2}$ at $37^{\circ} \mathrm{C}$. Digestion and passage were performed every 2-3 days using $0.05 \%$ trypsin (GIBCO, ThermoFisher Scientific, Inc.) and $0.01 \%$ EDTA (Sigma Chemical) (9).

Cytotoxic drugs. The chemotherapy drugs paclitaxel and carboplatin were obtained from the hospital pharmacy of the University Clinic Tianjin, and stored at $4^{\circ} \mathrm{C}$. Drugs were dissolved prior to use in RPMI-1640 medium (Invitrogen; Thermo Fisher Scientific, Inc.) without FBS or antibiotics.

siRNA transfection. A total of $500 \mu \mathrm{l}$ of OVCAR3 cell suspension at $1.4 \times 10^{5}$ cells $/ \mathrm{ml}$ were seeded in each well of a $24-w e l l$ dish. The subsequent day, if the cells reached $50 \%$ confluence, according to the manufacturer's directions, 60-100 pmol STK17A/siRNA (pSilencer/STK17A group)or negative siRNA (pSilencer group) were added with oligofectamine (Invitrogen, ThermoFisher Scientific, Inc.). Cells were harvested $72 \mathrm{~h}$ or 5 days later for semi-quantitative reverse transcription-polymerase chain reaction (RT-PCR) and western blot analysis, respectively (10). Negative siRNA transfected cells were used as negative control, while untransfected cells served as blank control. The sequences of STK17A siRNA were designed as follows: Sense, 5'-GATCCCGCAGCAGGAGGAATATCT ACCTGTTTCAAGAGAACAGGTAGATATTCCTCCTGC TGTTTTTTGGAAA-3' and anti-sense, 5'-AGCTTTTCC AAAAAACAGCAGGAGGAATATCTACCTGTTCTCTTG AAACAGGTAGATATTCCTCCTGCTGCGG-3'. And the negative control siRNA sequences were: Sense, 5'-UUCUCC GAACGUGUCACGUTT-3' and anti-sense, 5'-ACGUGACAC GUUCGGAGAATT-3'. Both siRNA were synthesized by GenePharma Co. (Shanghai, China).

Amplification of STK17A gene and vector construction. The cDNA of human STK17A was generated using PCR, with HEK293 cell cDNA as a template. HEK293 cells were obtained from the TianJin Life Science Research Center (TianJin, China). The sequences of the primers containing EcoRI and XhoI restriction enzyme sites were as follows: Sense, 5'-CCTGAATTCCATGATCCCTTTGGAGAAG-3' and anti-sense, 5'-CTCCTCTCGAGTCTTGTAGCAAAG GTTCCTC-3'. PCR was performed using 12.5 pmol each primer, $5 \mathrm{u}$ Pfu DNA polymerase, $25 \mu \mathrm{M}$ of each dNTP and $1 \mathrm{X}$ Pfu polymerase buffer in a $50 \mu \mathrm{l}$ volume (Stratagene, La Jolla, CA, USA). The thermal profile for the PCR was: $5 \mathrm{~min} 95^{\circ} \mathrm{C}$ for denaturation, $45 \sec 95^{\circ} \mathrm{C}, 1 \min 60^{\circ} \mathrm{C}, 1 \min 72^{\circ} \mathrm{C}$, for a total of 30 cycles, and a final extension at $72^{\circ} \mathrm{C}$ for $10 \mathrm{~min}$. The PCR products were cloned into the plasmid pCDNA3flu, which has the C-terminal modified with three continuous hemaglutinin (HA) coding region, and was constructed in our laboratory. Sequence analysis was performed to verify the accuracy of the plasmid, which was named pCDNA3flu/STK17A. STK17A expression was subsequently assessed in pCDNA3flu/STK17A transfected cells by semi-quantitative RT-PCR and western blot analysis (10-13). Empty pCDNA3flu transfected cells were used as negative control, while untransfected cells served as blank control.

Semi-quantitative RT-PCR analysis. Cells were transfected with siRNA or pCDNA3flu for $48 \mathrm{~h}$ and the level of STK17A expression was measured by a two-step semi-quantitative RT-PCR. TriZOL was used to extract total RNA, then $5 \mu \mathrm{g}$ RNA was combined with $2.5 \mathrm{nmol}$ dNTPs, 50 pmol oligo-dT and $\mathrm{H}_{2} \mathrm{O}$, and preheated $2 \mathrm{~min}$ at $65^{\circ} \mathrm{C}$ for denaturation. The reaction was cooled to $4^{\circ} \mathrm{C}$, and then $4 \mu 15$ X RT Buffer, $10 \mathrm{mM}$ DTT, 200 u MMLV Reverse Transcriptase and $40 \mathrm{u}$ RNAsin (Promega Corporation) were added resulting in a volume of $20 \mu \mathrm{l}$. The RT mix was incubated at $42^{\circ} \mathrm{C}$ for $60 \mathrm{~min}$, then $70^{\circ} \mathrm{C}$ for $10 \mathrm{~min}$. The cDNA was stored at $-20^{\circ} \mathrm{C}$. The yield of cDNA was measured according to the STK17A PCR signal generated relative to the internal standard $\beta$-actin (10). Primer sequences of $\beta$-actin gene were as follows: Sense, 5'-CGTGACATTAAG GAGAAGCTG-3' and anti-sense, 5'-CTAGAAGCATTTGCG GTGGAC-3'. The STK17A gene primer sequence is as above. The thermal profile for the $\mathrm{PCR}$ reaction included 26 cycles as follows: $95^{\circ} \mathrm{C}$ for $45 \mathrm{sec}, 60^{\circ} \mathrm{C}$ for $1 \mathrm{~min}, 72^{\circ} \mathrm{C}$ for $1 \mathrm{~min}$, using 5 U Taq Polymerase (Fermantas, ThermoFisher Scientific, Inc.) in a $50 \mu \mathrm{l}$ volume. The samples of PCR were run on $1 \%$ polyacrylamide gels, then analyzed using the labwork software, version 4.0 (UVP, Upland, CA, USA). All the primers were synthesized by the Sunbiotech Inc (Beijing, China).

Western blot analysis. Cells were transfected for $48 \mathrm{~h}$, prior to the addition of cell lysis buffer to extract proteins. The homogenate was then centrifuged at $700 \mathrm{x}$ g for $5 \mathrm{~min}$ to pellet large cellular debris. The samples were heated at $100^{\circ} \mathrm{C}$ for 5 min then loaded onto 15\% SDS-PAGE for electrophoresis. For the western blot analysis, the separated proteins were transferred to Protran nitrocellulose (Micron separations, Inc, Westborough, MA, USA). The blots were blocked in blocking buffer [ $1 \%$ phosphate-buffered saline (PBS), $0.1 \%$ Tween 20 and $3 \%$ bovine serum albumin) and then incubated with rabbit anti-STK17A antibody (SRP00679; Saier Corporation, Tianjin, China) at a 1:100 dilution, $\gamma$-tubulin acted as an internal standard that detected by mouse anti $\gamma$-tubulin (sc-51715; Santa Cruz Biotechnology, Inc., Dallas, TX, USA) at 1:500 dilution. The primary antibodies were detected with secondary goat anti-rabbit IgG (diluted 1:2,000 in PBS containing Tween 20; A0545; Sigma-Aldrich) conjugated with horseradish peroxidase (Sigma-Aldrich). Labeled bands from the blots were detected by enhanced chemiluminescence (Beyotime Institute of Biotechnology, Haimen, China ). The bands intensities were quantified by LabWorks software 4.0 as previously described (10).

Cell proliferation and apoptosis assays. Flow cytometry was used to analyze the cell cycle. After the cells were transfected for $48 \mathrm{~h}$, they were then digested with trypsin and resuspended in complete medium. Cells were centrifuged at 15,000 x g for 
$5 \mathrm{~min}$ and then resuspended in $1 \mathrm{ml}$ of PBS, then centrifuged again at $15,000 \mathrm{x} \mathrm{g}$ for $5 \mathrm{~min}$ to pellet the cells. The cells were washed in $400 \mu \mathrm{l}$ PBS and fixed in $4.5 \mathrm{ml} 85 \%$ ice-cold ethanol. Cells were pelleted by centrifuging at $15,000 \mathrm{x} \mathrm{g}$ for $5 \mathrm{~min}$, then resuspended in $600 \mu \mathrm{l} \mathrm{PBS}$ containing $2 \mathrm{mg} / \mathrm{ml}$ propidium iodide and $1 \mathrm{mg} / \mathrm{ml}$ RNase A (Sigma-Aldrich). A flow cytometer (BD Biosciences, San Jose, CA, USA) was used to determine the cell cycle profiles and the results were analyzed with ModFIT software (Verity Software House, Inc., Topsham, ME, USA). Cells were incubated in a solution of $0.5 \mathrm{~g} / \mathrm{ml}$ FITC-labeled annexin V (Roche Diagnostics, Nutley, NJ, USA) after washing with PBS. The proportion of dead cells were assessed by the PI exclusion method and flow cytometry, as previously described $(9,14)$.

Cell growth curve and doubling time. A single-cell suspension was achieved by trypsinization. An equal number of cells $\left(1 \times 10^{4}\right)$ were seeded into each well of a 24 -well plate in $10 \%$ fetal bovine serum supplemented RPMI-1640 medium. The cell lines were cultured in a humidified incubator atmosphere containing $5 \% \mathrm{CO}_{2}$ at $37^{\circ} \mathrm{C}$ and the culture medium was replaced every 2 days. The cells of three wells were counted manually every $24 \mathrm{~h}$ for 7 days, as previously described $(10,15)$. Cell doubling time was calculated by the Patterson formula:

$$
T_{\mathrm{D}} 280=T \times \log 2 /\left(\log N_{\mathrm{t}}-\log N_{0}\right)(15) .
$$

MTT assay. The level of resistance toward chemotherapeutic drug was examined using the MTT assay. Cells transfected with STK17A/siRNA or pCDNA3flu/STK17A were assessed, using negative siRNA or empty pCDNA3flu transfected cells as controls. Following transfection for $72 \mathrm{~h}$, the cells were re-seeded in triplicate at a density of $\sim 6 \times 10^{3}-8 \times 10^{3}$ cells/well in 96-well dishes in $100 \mu \mathrm{l}$ complete medium for $24 \mathrm{~h}$. Then paclitaxel or carboplatin, at various concentrations were added to the medium. After $48 \mathrm{~h}, 2 \mathrm{mg} / \mathrm{ml}$ MTT (Sigma-Aldrich) was added to each well, then incubated for an additional $4 \mathrm{~h}$ at $37^{\circ} \mathrm{C}$. The plates were centrifuged at $500 \mathrm{x}$ g for $10 \mathrm{~min}$. The medium was removed from each well, and $100 \mu 1$ of DMSO (Sigma-Aldrich) was added, and the plates were shaken for $5 \mathrm{~min}$. The absorbance was detected by a micro-plate reader at a wavelength of $570 \mathrm{~nm}$. Cell survival rate was calculated using the following formula: 1-(mean absorbance of untreated wells-mean absorbance of 3 test wells)/(mean absorbance of untreated wells-mean absorbance of 3 medium control wells) $(15,16)$. The $50 \%$ inhibitory concentration $\left(\mathrm{IC}_{50}\right)$ was to evaluate the value $(14,17)$.

Statistical analysis. Statistical significance was calculated using the independent samples $t$-test and one way analysis of variance with the statistical software package SPSS (SPSS Inc., Chicago, IL, USA). $\mathrm{P}<0.05$ was considered to indicate a statistically significant difference (18).

\section{Results}

OVCAR3/STK17A plasmid construction and inhibition of STK17A by siRNA. Western blot analysis demonstrates that STK17A was expressed in OVCAR3 cells. The STK17A gene was cloned into the plasmid pCDNA3flu, which has the
C-terminal modified with three continuous HA coding regions. After transfection with pCDNA3flu/STK17A for $48 \mathrm{~h}$, the level of STK17A mRNA expression was increased 3 -fold. Actin acted as a loading control to normalize the level of STK17A expression (Fig 1A). Increased level of STK17A protein was also significant in the OVCAR3/STK17A transfected cells (Fig. 1B).

Semi-quantitative RT-PCR and western blotting were used to examine the efficiency of STK17A targeted siRNA-mediated down-regulation of STK17A. After transfection for $48 \mathrm{~h}$, the levels of STK17A expression were distinct between transfected and untransfected cells (Fig. 1C). Decreased levels of STK17A protein were significant in the STK17A/siRNA transfected cells. $\gamma$-tubulin acted as a loading control to normalize the levels of STK17A expression (Fig. 1D).

\section{Cell proliferation and apoptosis assays}

Flow cytometric analysis. After stimulating for $8 \mathrm{~h}$ using annexin-V and propidium iodide (PI) staining, the cells transfected with STK17A/siRNA and negative siRNA were analyzed by flow cytometry and demonstrated an approximately equal apoptotic pattern, similar to that of untransfected cells, evidenced as annexin-V-positive and PI-negative (Fig. 2A). The apoptotic percentage (annexin-V-positive, PI-negative, lower right quadrant) was $0.91 \%$ for STK17A/siRNA, $0.65 \%$ for negative siRNA and $0.85 \%$ for untransfected cells, which confirmed that STK17A could not induce apoptosis in OVCAR3 cells. When the cell cycle was analysed by PI staining, the G2 population of cells was $28.91 \%$ for the STK17A/siRNA transfection group, $18.86 \%$ for the negative siRNA transfection group and $19.66 \%$ for untransfected cells (Fig. 2B). Thus, the cells from the STK17A/siRNA transfection group demonstrated an increased percentage of $\mathrm{G} 2$ phase cells. To better characterize the effect of STK17A on reducing cell proliferation, growth curves were produced using STK17A/siRNA transfected cells and negative siRNA transfected cells maintained in the presence of $10 \%$ FBS. As shown by the cell doubling time results, there was a marked improvement in the proliferative response of STK17A/siRNA transfected cells. This enhanced effect became evident at day 6 , as the cells reached confluence. The mean of results from three independent experiments indicated that there was a $45.0 \pm 3.4 \%$ increase in cell number at day 7 after seeding in STK17A/siRNA cells compared with negative siRNA transfected cells. In addition, the antiproliferative effect of STK17A was observed in the pCDNA3flu/STK17A transfected cells, where there was a $39.0 \pm 5.4 \%$ reduction in cell number at day 7 compared with the empty pCDNA3flu transfected control cells (Fig. 3). The results yielded a similar tendency to the data from the flow cytometric analysis.

Drug resistance assays. After treatment with carboplatin or paclitaxel for $72 \mathrm{~h}$, dose-response curves were calculated using MTT survival assays of cells transfected with pCDNA3flu/STK17A or empty pCDNA3flu. pCDNA3flu/STK17A transfected cells showed enhanced drug-sensitivity to carboplatin and paclitaxel. The differences were significant at carboplatin concentrations of 3,5, 10 and $20 \mu \mathrm{g} / \mathrm{ml}$, and at the paclitaxel concentrations of $0.6,3,6$ and $12 \mu \mathrm{g} / \mathrm{ml}$ (Wilcoxon signed-rank test, $\mathrm{P}<0.05$ ). The $\mathrm{IC}_{50}$ of the two drugs in cells were calculated from the curves, and it was determined that the $\mathrm{IC}_{50}$ value of carboplatin and paclitaxel decreased 1.45-fold and 1.56-fold, respectively, in 


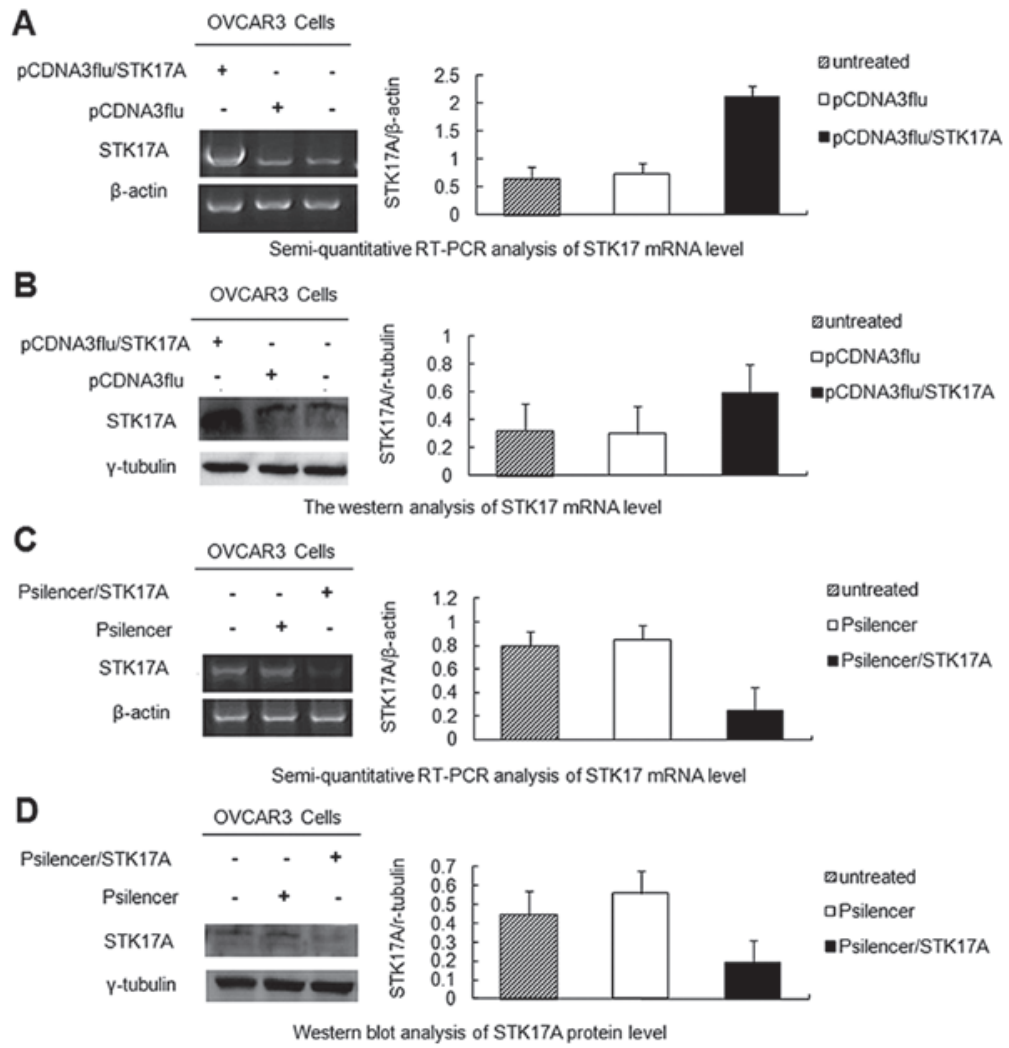

Figure 1. STK17A RT-PCR and western analysis. Both the RNA and protein level demonstrated that the designed STK17A/siRNA or pCDNA3flu/STK17A effectively induced the target gene degradation and up-regulation. (A) The STK17A mRNA up-regulation was 3-fold increased compared with the control in the pCDNA3flu/STK17A transfected cells. (B) High STK17A expression in the pCDNA3flu/STK17A transfected cells was also observed by western blotting. (C) The STK17A mRNA degradation degree is $\sim 2-3$ fold compared with the control in the STK17A/siRNA transfected cells. The distinction was significant at $48 \mathrm{~h}$ after initial transfection by semi-quantitative RT-PCR and (D) protein was similarly reduced as assessed by western blot analysis. RT-PCR, reverse transcription-polymerase chain reaction; siRNA, small interfering RNA; mRNA, messenger RNA.

A Annexin-V \& propidium iodide
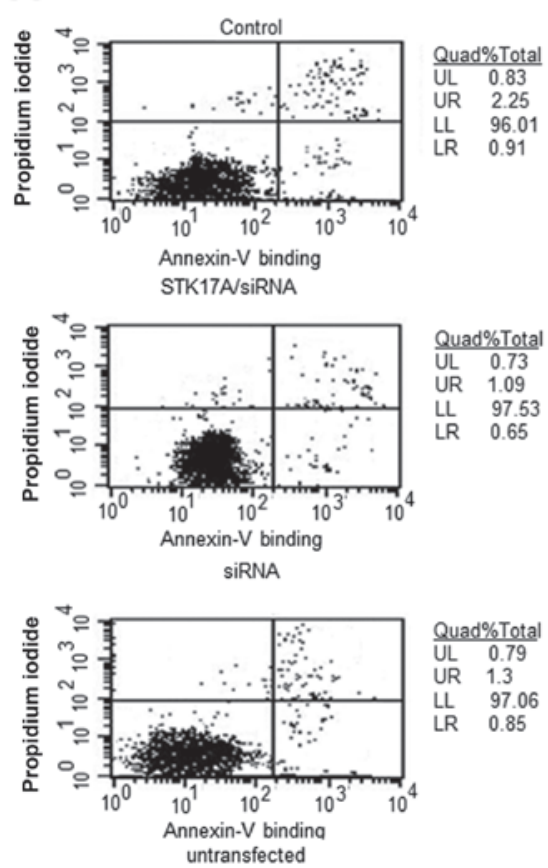

B Permeabilized propidium iodide

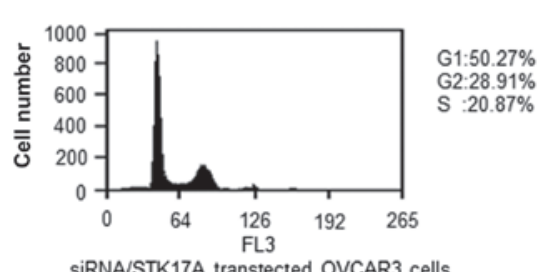

siRNAVSTK17A transtected OVCAR3 cells

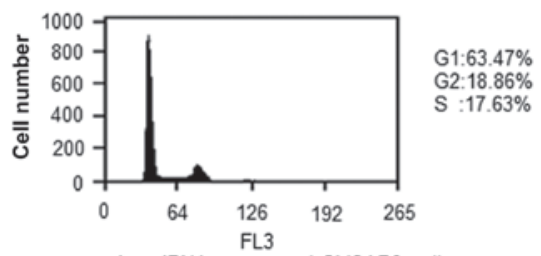

negative siRNA transtected OVCAR3 cells

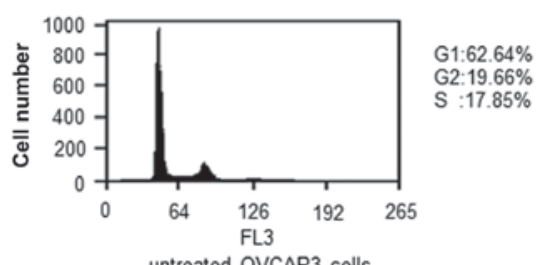

Figure 2. Flow cytometric analysis. The cells transfected with STK17A/siRNA and negative siRNA showed an approximately equal apoptotic pattern, similar to that of untreated cells as shown by (A) annexin-V-positive and PI-negative. The percentage of cells showing an apoptotic pattern (annexin-V-positive, PI-negative, lower right quadrant) was $0.91 \%$ for STK17A/siRNA, $0.65 \%$ for negative siRNA and $0.85 \%$ for untransfected cells. (B) When the cell cycle was analyzed by PI staining, the G2 population of cells was $28.91 \%$ for the STK17A/siRNA transfection group, $18.86 \%$ for the negative siRNA transfection group and $19.66 \%$ for the untransfected cells (untreated OVCAR3 cells in panel B). Thus, the STK17A/siRNA transfected cells showed an increased percentage of replicated DNA; siRNA, small interfering RNA; PI, propidium iodide. 

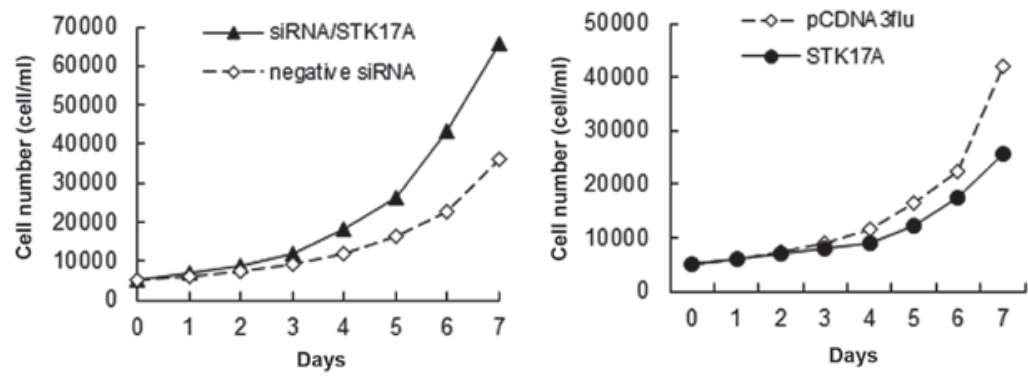

Figure 3. Cell growth. The cell doubling times of STK17A/siRNA transfected cells and controls were 45.19 and 58.89 h, respectively, while those of pCDNA3flu/STK17A transfected cells and controls were 54.64 and $71.34 \mathrm{~h}$, respectively. siRNA, small interfering RNA.
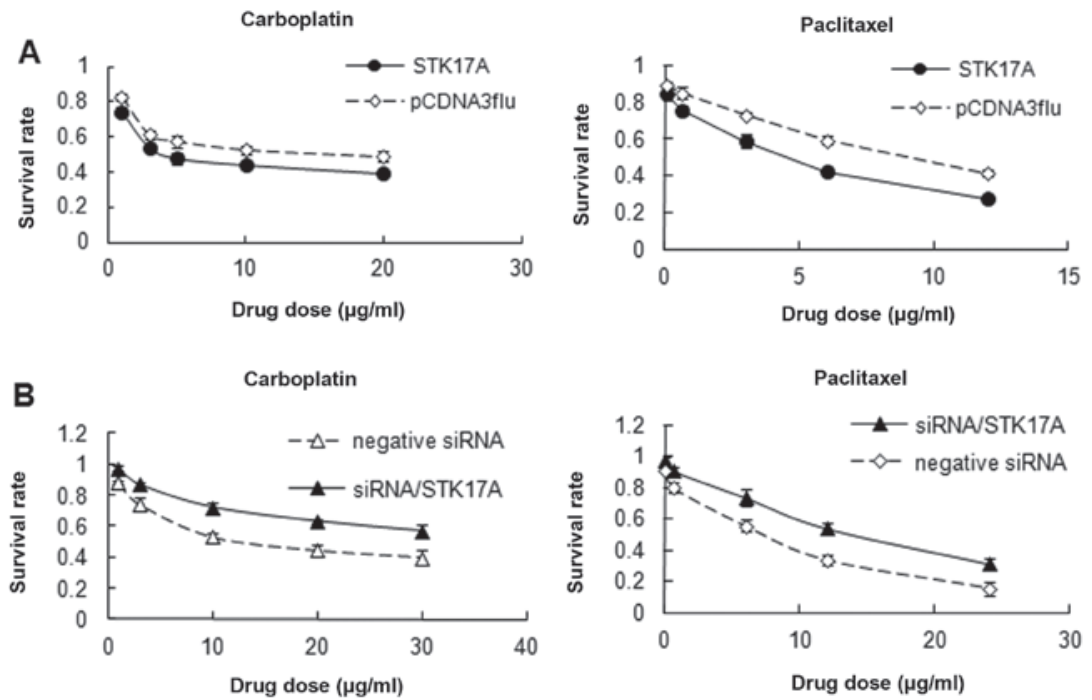

Figure 4. MTT analysis. (A) Overexpression of STK17A enhances cell sensitivity to carboplatin and paclitaxel. Being incubated for $24 \mathrm{~h}$ in complete media supplemented with $10 \%$ FBS, cells were incubated with carboplatin or paclitaxel for $48 \mathrm{~h}$. Then MTT was added to assay the survival rate (P<0.05, Wilcoxon test). (B) Inhibition of STK17A enhanced drug resistance in OVCAR3 cells. Cells were seeded 96-well plates $48 \mathrm{~h}$ after transient transfection with siRNA. Being incubated for $24 \mathrm{~h}$ in complete media supplemented with $10 \%$ FBS, cells were challenged with fresh media containing different dilutions of each drug. After incubation with carboplatin or paclitaxel for $48 \mathrm{~h}$ MTT was added to assay the survival rate. Inhibition of STK17A messenger RNA and protein expression increased resistance of OVCAR3 cells to paclitaxel (curve labeled 'siRNA/STK17A' in B) $(\mathrm{P}<0.05$, Wilcoxon test) and carboplatin ( $\mathrm{P}<0.05$, Wilcoxon test). Error bars represent the standard deviation for 3 independent experiments. siRNA, small interfering RNA.

pCDNA3flu/STK17A transfected cells. The above data indicate that overexpression of STK17A may enhance the sensitivity of cells to carboplatin and paclitaxel. After the cells were treated with paclitaxel or carboplatin for 3 days, the survival rate of OVCAR3 cells was increased compared with the control (Wilcoxon signed-rank test, $\mathrm{P}<0.05$ ). Dose-response curves from MTT survival assays demonstrated a difference in survival rates (Fig. 4). Consistent with the above findings, inhibition of STK17A mRNA and protein expression increased resistance of OVCAR3 cells to paclitaxel $(\mathrm{P}<0.05)$ and carboplatin $(\mathrm{P}<0.05)$. Taken together, these data provided evidence that inhibition of STK17A may increase resistance to paclitaxel and carboplatin in ovarian cancer OVCAR3 cells.

\section{Discussion}

The present study demonstrated that up-regulation of STK17A may enhance the sensitivity of OVCAR3 cells to carboplatin and paclitaxel. This conclusion was further confirmed by the transfection of ovarian cancer cells with STK17A-targeted siRNA. Based upon these data, we propose that STK17A may act as a key factor in the drug-resistance phenotype of ovarian cancer cells.

It has previously been established that postoperative treatment of ovarian cancer is mostly chemotherapy with carboplatin combined with paclitaxel. However, the drug-resistance of cells often leads to treatment failure. Mechanisms have been proposed for the resistance to paclitaxel in ovarian cancers, such as single nucleotide polymorphisms (SNPs) in the gene ABCB1, which encodes the transport protein, P-glycoprotein (19) and mutations in the target protein b-tubulin (20). Certain mechanisms of drug resistance are known, including cytoplasmic detoxification in response to platinate and increased DNA repair in response to carboplatin $(1,19)$. A number of studies have identified that p53 and mdr-1 have higher levels of expression in ovarian cancer cells that recovered from platinate agent and paclitaxel treatments $(19,20)$.

STK17A is thought to act primarily causing apoptosis in response to various apoptotic stimuli as TNF-a, FasL, UV light, and certain drugs. Overexpression of STK17A induces the morphological changes of apoptosis in NIH 3T3 mouse embryonic fibroblast cells. However, overexpression of 
STK17A did not induce apoptosis in COS7 African green monkey kidney cells (7) similarly in OVCAR3 cells. Recently it was demonstrated that STK17A was down regulated in SK-NEP-1 cells (Wilms' tumor cell line) treated with YM155 (an inhibitor of survivin) compared with DMSO control group by Ingenuity pathway analysis (21). One hypothesis is that the genetic changes that protect cells from STK17A-induced apoptosis may have occurred in the immortalization process of COS7 and OVCAR3 cells or that the sensitivity to STK17A-induced apoptosis is different depending on the cell type. STK17A has also been associated with cross-resistance against DNA-damaging drugs in human malignant melanoma cell line MeWo cells, with oxaliplatin and 5-fluorouracil resistance in colon cancer cells (5). The expression of STK17A in various types of tumor cells and its roles in tumorigenesis and metastasis is not fully known.

In the present study, it was found that the overexpression of STK17A increases the cell proliferation, the doubling time of the cells was two-thirds of the controls. The number of cells in G2/M stage were increased and decreased in G1 stage, as demonstrated by flow cytometric assay. However, in the complex process of intrinsic and acquired drug resistance of cancer cells, STK17A maybe play some additional important roles.

In the future, studies may continue to identify the novel mechanisms of drug resistance, that would enable the development of a novel generation of anti-cancer drugs, enhancing the drug-sensitivity of cancer cells.

The present study demonstrates that STK17A acts as a key role in the drug-resistance phenotype of ovarian cancer cells. Variable susceptibility to carboplatin, paclitaxel results from different expression levels of STK17A in OVCAR3 cells. The growth of STK17A/siRNA transfected cells was promoted compared with the controls, accordingly the cell doubling time was shortened.

\section{Acknowledgements}

The authors thank Mr. Yixuan Li, Mr. Fang Wang and Mr. Juan Ge (Tianjin Life Science Center, Tianjin Medical University) for their technical assistance. Financial support comes from the Youth Science Foundation of Heilongjiang Province (grant no. QC2009C28).

\section{References}

1. Zhang WB: Ovarian cancer chemotherapy drug resistance related gene and the research progress of prognosis. Foreign Medical Gynecology Pathol 34: 193-196, 2007.
2. Liao Y and Qi L: Advances in chemotherapy resistance in ovarian clear cell carcinoma of molecular biology. International Journal of Obstetrics and Gynecology 34: 69-72, 2007.

3. Gottesman MM,Fojo T and Bates SE: Multidrug resistance in cancer: Role of ATP-dependent transporters. Nat Rev Cancer 2: 48-58, 2002.

4. Zhang YL: The family of copper ion transporters and ovarian cancer research progress of relationship between cisplatin resistance. Prog Obstet Gynecol 16: 388-390, 2007.

5. Tang H, Liu YJ, Liu M and Li X: Establishment and gene analysis of an oxaliplatin-resistant colon cancer cell line THC8307/L-OHP. Anticancer Drugs 18: 633-639, 2007.

6. Wittig R, Nessling M, Will RD, Mollenhauer J, Salowsky R, Münstermann E, Schick M, Helmbach H, Gschwendt B, Korn B, et al: Candidate genes for cross-resistance against DNA-damaging drugs. Cancer Res 62: 6698-6705, 2002.

7. Sanjo H, Kawai T and Akira S: DRAKs, novel serine/threonine kinases related to death-associated protein kinase that trigger apoptosis. J Biol Chem 273: 29066-29071, 1998.

8. Wang Q, He Z, Gao J, Hu S, Huang M, Liu M, Zheng J and Tang H: S100P sensitizes ovarian cancer cells to carboplatin and paclitaxel in vitro. Cancer Lett 272: 277-284, 2008.

9. Gao JT: Effects of cyclosporine a or tacrolimus combined with epirubicin on the proliferation and apoptosis of human hepatocellular carcinoma cell lines. Master Degree Thesis of Zhejiang University, 2006.

10. Wang DQ: Inhibition of HPV16 E7 oncogene in cervical cancer by E7-specific siRNA expression vector. PhD Thesis of Sichuan University, 2005.

11. Cao MM: Screening for the signature profile of cisplatin resistance in human ovarian cancer by cDNA microarray. $\mathrm{PhD}$ Thesis of First Military Medical University, 2005.

12. Hu XM: Cloning and immunoprotection of schistosoma japonicum (Chinese Mainland Strain) mitochondria related protein and its antigenic epitopes. PhD Thesis of Nanjing Medical University, 2001.

13. Gai XD: Study on the reversal of multidrug resistance of human hepatocellular carcinoma and related mechanisms. $\mathrm{PhD}$ Thesis of Jilin University, 2004.

14. Xu YP: Iressa (ZD1839) radiation effect of A549 lung cancer cell line, zhejiang university, master's degree thesis, 2006.

15. Zhao H: The experiment of the advancing chemosensitization by triiodotyronone (T3) and Insulin. China PLA Postgraduate Medical School Master's Degree Thesis, 2002.

16. Xiong J, Peng ZL and Tan X: Effects of adenoviral-mediated mda-7/IL-24 gene infection on the growth and drug-resistance of drug-resistant. Sichuan Da Xue Xue Bao Yi Xue Ban 38: 433-436, 2007 (In Chinese).

17. Shi J: MTX in different cancer patients with traditional Chinese medicine studies and individualized dosage regimen design. Master Degree Thesis of Qiingdao University, 2001.

18. Liu GY: Study on the machanisms responsible for cisplatin resistance and the reversal strategy in ovarian epithelial carcinoma. PhD Dissertation of Tianjin Medical University, 2004.

19. Jiang J: P53 gene's relationship with ovarian cancer drug resistance. Practical Medical Journal 23: 1588-1590, 2007.

20. Pan S, Wu XF and Chen HZ: Relationship of cyclooxygenase-2 expression and chemotherapy resistance, prognosis of patients with epithelial ovarian cancer. Tumor 27: 46-49, 2007.

21. Tao YF, Lu J, Du XJ, Sun LC, Zhao X, Peng L, Cao L, Xiao PF, Pang L, Wu D, et al: Survivin selective inhibitor YM155 induce apoptosis in SK-NEP-1 Wilms tumor cells. BMC Cancer 12: 619, 2012. 